\title{
Complete Reconstitution and Deorphanization of the 3 MDa NOCAP (NOCardiosis-Associated Polyketide) Synthase
}

\author{
Kai P. Yuet ${ }^{\dagger}$, Corey W. Liu ${ }^{\ddagger}, \|, \perp$, Stephen R. Lynch ${ }^{\dagger, \perp}$, James Kuo ${ }^{\boldsymbol{\Phi}, \#, ~ W e s l e y ~ M i c h a e l s ~}{ }^{\top}$, Robert B.

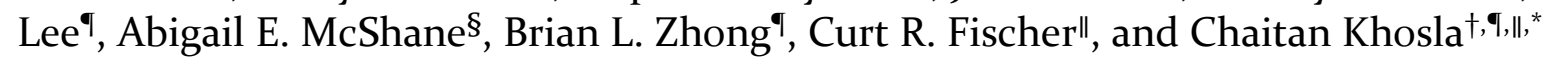 \\ ${ }^{\dagger}$ Department of Chemistry, ${ }^{\ddagger}$ Department of Structural Biology, `Department of Chemical Engineering, ${ }^{\S}$ Department \\ of Bioengineering, "Stanford ChEM-H, Stanford University, Stanford, CA 94305
}

\begin{abstract}
Several Nocardia strains associated with nocardiosis, a potentially life-threatening disease, house a nonamodular assembly-line polyketide synthase (PKS) that presumably synthesizes an unknown natural product. Here, we report the discovery and structure elucidation of the NOCAP (NOCardiosis-Associated Polyketide) aglycone by first fully reconstituting the NOCAP synthase in vitro from purified protein components followed by heterologous expression in $E$. coli and spectroscopic analysis of the purified products. The NOCAP aglycone has an unprecedented structure comprised of a substituted resorcylaldehyde headgroup linked to a 15-carbon tail that harbors two conjugated all-trans trienes separated by a stereogenic hydroxyl group. This report is the first example of reconstituting a trans-acyltransferase assemblyline PKS either in vitro or in E. coli, and of using these approaches to "deorphanize" a complete assembly-line PKS identified via genomic sequencing. With the NOCAP aglycone in hand, the stage is set for understanding how this PKS and associated tailoring enzymes confer an advantage to their native hosts during human Nocardia infections.
\end{abstract}

Within the past decade, genomic sequencing has exposed many "orphan" biosynthetic gene clusters encoding assembly-line PKSs whose products have yet to be identified $^{1}$. Analysis of orphan PKSs has the potential to reveal new biosynthetic strategies as well as products with unprecedented structures and biological activities. Of particular interest to our laboratory is an intriguing family of orphan assembly-line PKSs termed NOCAP synthases. NOCAP synthases harbor cis- and trans-acyltransferases ${ }^{2}$, and are invariably found in strains of the actinomycete Nocardia isolated from patients affected with nocardiosis, a serious pulmonary or systemic disease ${ }^{-5}$ (Table $\mathbf{S}_{\mathbf{1}}$ ). The NOCAP synthase is composed of four separate proteins (NOCAP_PKS1-4) containing nine PKS modules (eight of which are collinear) (Figure 1). Modules 1 and 3 possess their own acyltransferase domains, whereas the remaining modules require a trans-acyltransferase ( $t$ AT) to supply malonyl extender units. Notably, this PKS has three other unusual features: a) a "split and stuttering" module capable of catalyzing three elongation and reductive cycles (module 5) 5,8,9, b) a terminal thioester reductase $(\mathrm{TR})^{10}$, and c) a thioesterase (TE) domain fused to the $t \mathrm{AT}$. In a preliminary study, several unprecedented, albeit partially characterized, octaketide and heptaketide products were generated by incubating purified modules $4-8$ with the surrogate primer unit octanoyl-CoA5. Building on our laboratory's prior experience in functionally reconstituting the complete 6-deoxyerythronolide B synthase (DEBS) in $E$. coli ${ }^{6}$ and from purified protein components 7 , we sought to deorphanize a prototypical NOCAP synthase outside of its genetically difficult and potentially hazard- ous natural host using both of these approaches. Here we report on the successful reconstitution of the entire assembly-line NOCAP synthase in vitro as well as in E. coli.

We hypothesized that the uncharacterized module "X" synthesizes a primer unit for the collinear assembly line comprised of modules 1-8. Accordingly, we expressed a soluble maltose-binding protein (MBP)-module $\mathrm{X}$ fusion protein in E. coli, and purified it to homogeneity (Figure S1). To identify the substrates and products bound to its acyl carrier protein (ACP) domain by intact protein LCMS, we further expressed and purified two derivatives of this protein: MBP-module $\mathrm{X}$ without the ACP domain $\left(\mathrm{KS}_{\mathrm{X}}\right)$, and the stand-alone $\mathrm{ACP}_{\mathrm{X}}$ (Figure S2). Apo-ACPX was incubated with the Sfp phosphopantetheinyl transferase $^{11}$ and malonyl-CoA to obtain malonyl-S-ACPX, which was then incubated with $\mathrm{KS}_{\mathrm{x}}$. LC-MS analysis revealed that malonyl-S-ACPX was predominantly decarboxylated to acetyl-S-ACP ${ }_{\mathrm{x}}$ in a $\mathrm{KS}_{\mathrm{X}}$-dependent manner (Figure 2a), suggesting that $\mathrm{KS}_{\mathrm{X}}$ is condensationincompetent (hence the designation $\mathrm{KS}_{\mathrm{o}}$ from here onwards) but is able to decarboxylate malonyl-S-ACPx to generate an acetyl unit for translocation to module 1 . Interestingly, while $\mathrm{KS}_{\mathrm{X}}$ appears functionally analogous to specialized $\mathrm{KS}_{\mathrm{Q}}$ domains $\mathrm{s}^{12}$, its active site Cys residue is not replaced by a highly conserved Gln. $\mathrm{KS}_{0}$ domains are prevalent in trans-AT PKSs, and lack the active site His residue located within the HGTGT motif ${ }^{2}$; however, $\mathrm{KS}_{\mathrm{X}}$ also retains this conserved His residue. 
a

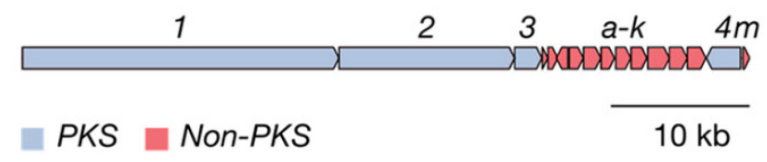

b

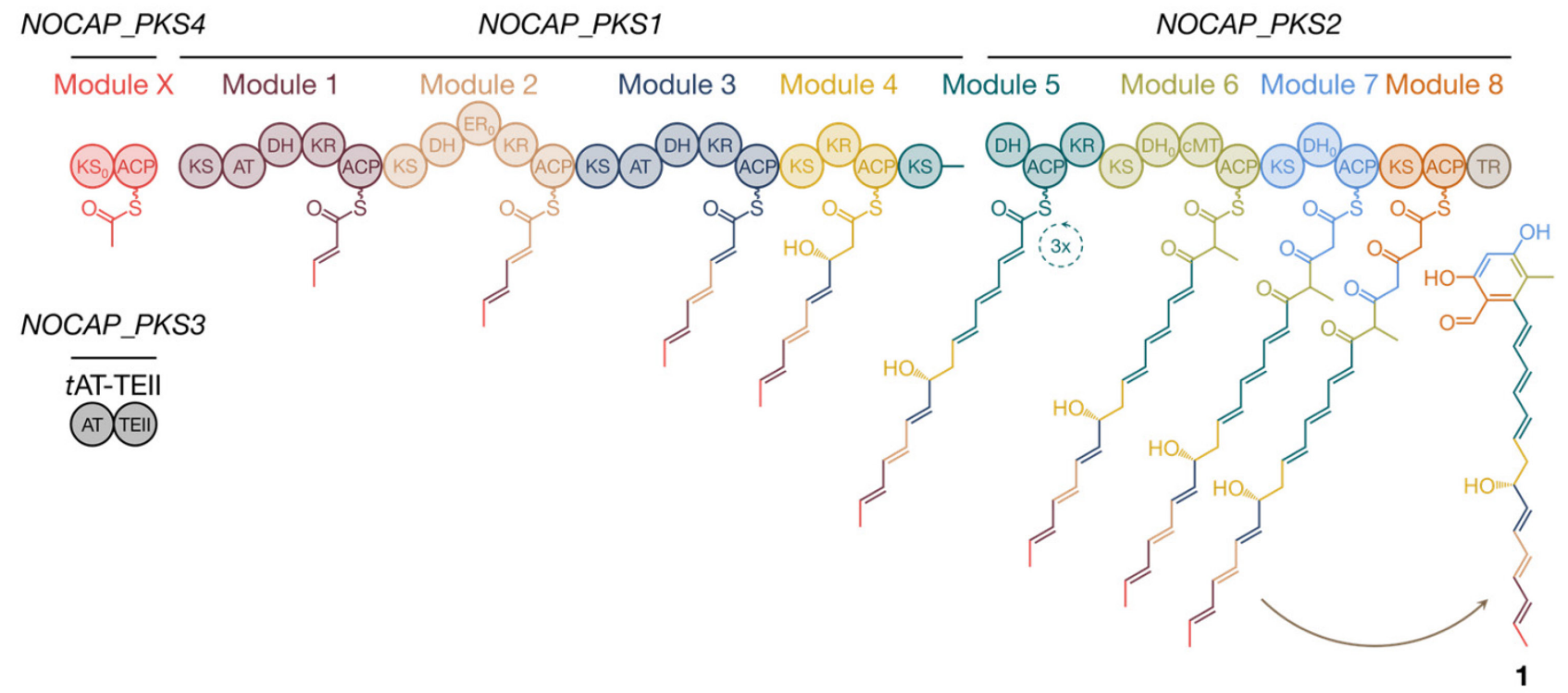

Figure 1. (a) Prototypical NOCAP synthase biosynthetic gene cluster from N. puris. (b) Biosynthesis of $\mathbf{1}$ by the NOCAP synthase. Key: KS, ketosynthase; AT, acyltransferase; DH, dehydratase; KR, ketoreductase; ER, enoylreductase; cMT, $C$-methyltransferase; ACP, acyl carrier protein; TR, thioester reductase; and TEII, thioesterase II.

To assay the overall activity of modules $\mathrm{X}, 1$ and 2 , a bimodular protein $\left(\mathrm{KS}_{1}-\mathrm{AT}_{1}-\mathrm{DH}_{1}-\mathrm{KR}_{1}-\mathrm{ACP}_{1}-\mathrm{KS}_{2}-\mathrm{DH}_{2}-\mathrm{ER}_{2}-\right.$ $\mathrm{KR}_{2}$ ) lacking $\mathrm{ACP}_{2}$ was expressed and purified; separately, stand-alone holo- $\mathrm{ACP}_{2}$ was also expressed and purified (Figure $\mathrm{S}_{3}$ ). The two proteins were assayed via a phosphopantetheine (PPant) ejection assay ${ }^{13}$ in the presence of MBP-module X, truncated tAT (i.e., lacking its TE domain) and appropriate substrates. In this and all subsequent assays utilizing malonyl-CoA, this labile substrate was generated in situ by adding malonic acid, CoASH, ATP and the Streptomyces coelicolor malonyl-CoA synthetase MatB ${ }^{14}$ to the reaction mixture. Instead of detecting the anticipated hex-4-enoyl-PPant species, sorbylPPant was the major observed product (Figure $\mathbf{2 b}$ ), implying that the enoylreductase (ER) domain of module 2 is inactive (designated $\mathrm{ER}_{\mathrm{o}}$ from here onwards). Together, these results confirm our hypothesis that module $\mathrm{X}$ module 1-module 2 comprise the first three modules for initiation of NOCAP biosynthesis.

We hypothesized that the TE domain of the $t$ AT-TE protein hydrolyzes acyl-ACPs under conditions of "stalled" polyketide biosynthesis ${ }^{15}$. This suggestion was consistent with our earlier observation that use of the truncated $t$ AT in place of the full-length tAT-TE resulted in a two-toten-fold decrease in product formation' ${ }^{5}$. To test this hypothesis, we incubated Sfp-derived acetyl-S-ACP - a stalled acyl-ACP surrogate - with either $t$ AT-TE or $t$ AT. LC-MS analysis uncovered that acetyl-S-ACP1 was hydrolyzed to holo-ACP ${ }_{1}$ in the presence of $t \mathrm{AT}-\mathrm{TE}$ but not $t \mathrm{AT}$ (Figures 2c, S4). Analogous radiolabeling experiments further verified the above findings (Figure $\mathbf{S}_{5}$ ). Together, these results provide strong evidence that this TE is a member of the "TEII" sub-family of thioesterases (designated TEII hereafter) that acts as a proofreading enzyme by hydrolyzing unproductive intermediates.

Buoyed by the reconstitution of modules $\mathrm{X}, 1$ and 2, we endeavored to reconstitute in vitro the complete NOCAP synthase. To overcome its exceptionally large size (the synthase's homodimeric mass approaches $3 \mathrm{MDa}$ ), NOCAP_PKS1 was expressed and purified as three standalone proteins: modules 1 and 2 as one bimodular protein, module 3 as a unimodular protein, and module 4 along with the KS domain of module 5 (module $4-\mathrm{KS}_{5}$ ) as the third protein. Separately, NOCAP_PKS2 was dissociated into two proteins: the DH-ACP-KR tridomain of module 5 fused to the complete module $6\left(\mathrm{DH}_{5}-\mathrm{ACP}_{5}-\mathrm{KR}_{5}\right.$-module $6)$, and a bimodular protein comprised of modules 7 and 8 along with the terminal TR domain (modules 7-8-TR) (Figures 3a, S1). To facilitate intermodular chain translocation between separated modules, each protein was fused to complementary $\mathrm{N}$-terminal and/or C-terminal docking domains from DEBS that have previously been shown to facilitate non-covalent interactions between successive modules on a PKS assembly line ${ }^{16,17}$. These five NOCAP synthase-derived proteins were mixed with MBPmodule $\mathrm{X}, t \mathrm{AT}$-TEII, malonyl-CoA, NADPH and $S$ adenosyl methionine. To confirm that products originated from the assembly-line PKS, $\left[2^{-13} \mathrm{C}\right]-,\left[1,3^{-13} C_{2}\right]-$ or $\left[{ }^{13} C_{3}\right]-$ malonyl-CoA was used in place of malonyl-CoA in parallel reactions. By high-resolution $\mathrm{MS}$, we identified a polyketide 1 with a molecular formula of $\mathrm{C}_{23} \mathrm{H}_{26} \mathrm{O}_{4}$ (observed [M- 
$\mathrm{H}]^{-} \mathrm{m} / \mathrm{z}$ 365.1762, theoretical $[\mathrm{M}-\mathrm{H}]^{-} \mathrm{m} / \mathrm{z} 365.1753,2.5$ ppm) (Figure 3c).

a
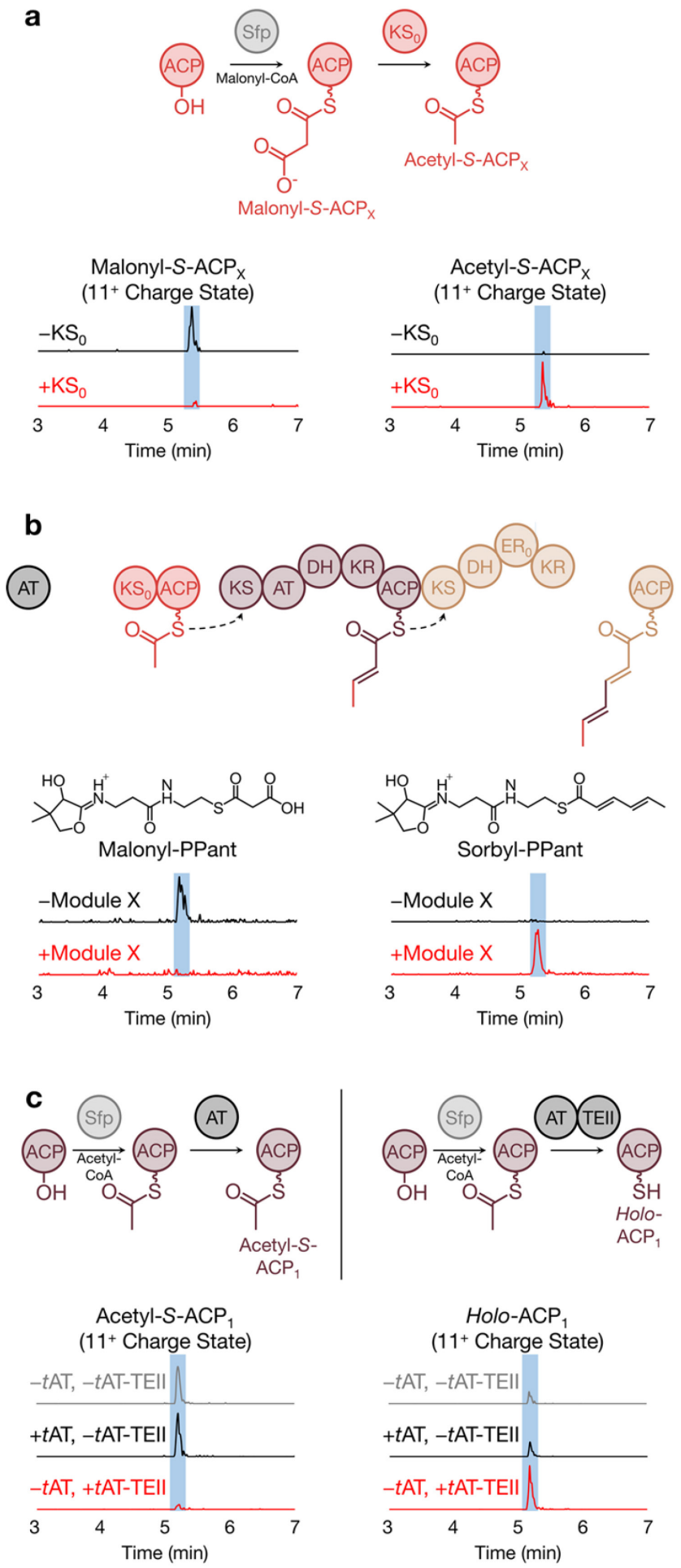

Figure 2. In vitro characterization of modules $\mathrm{X}, \mathrm{1-2}$ and $t \mathrm{AT}$ TEII. (a) Extracted ion chromatograms (EICs) of the $11^{+}$ charge states for malonyl-S-ACP and acetyl-S-ACP in the presence or absence of $\mathrm{KS}_{\mathrm{o}}$. (b) EICs of malonyl-PPant and sorbyl-PPant ejected from malonyl-S-ACP${ }_{2}$ and sorbyl-S$\mathrm{ACP}_{2}$, respectively, in the presence or absence of module $\mathrm{X}$. (c) EICs of the $11^{+}$charge states for acetyl-S-ACP${ }_{1}$ and holo$\mathrm{ACP}_{1}$ in the presence or absence of either $t \mathrm{AT}$ or $t \mathrm{AT}$-TEII.
The observation of $+11,+11$ and +22 mass shifts for 1 in mixtures containing $\left[2^{-13} \mathrm{C}\right],\left[1,3^{-13} \mathrm{C}_{2}\right]$ and $\left[{ }^{13} \mathrm{C}_{3}\right]$ malonylCoA, respectively, indicated that 1 traversed the entire polyketide synthase and underwent three rounds of chain elongation, ketoreduction and dehydration at module 5 (Figures 1, S6-7). Because biosynthesis of 1 requires the entire assembly-line PKS, we propose that $\mathbf{1}$ is the aglycone product of the NOCAP synthase. A closely related polyketide 2 was detected that had presumably undergone one fewer round of chain elongation, ketoreduction and dehydration than 1 (molecular formula $\mathrm{C}_{21} \mathrm{H}_{24} \mathrm{O}_{4}$, observed $[\mathrm{M}-\mathrm{H}]^{-} \mathrm{m} / \mathrm{z}$ 339.1604, theoretical $[\mathrm{M}-\mathrm{H}]^{-} \mathrm{m} / \mathrm{z}$ 339.1596, $2.4 \mathrm{ppm}$ ) (Figures S8-10). Its MS/MS fragmentation pattern matched well with that of $\mathbf{1}$, leading us to hypothesize that an upstream module was "skipped" during the biosynthesis of $\mathbf{2}$. Two more minor polyketides 3 and 4 were identified with MS/MS fragmentation patterns noticeably different than 1 and 2 (Figures S11-16). 3 and 4 are most likely premature polyketides with a pyrone moiety that originated from spontaneous release after module 7 extension and $\mathrm{C}-1-\mathrm{C}-5$ oxygen lactonization.

For definitive structural analysis, we sought to produce the NOCAP synthase products by using $E$. coli as a heterologous host for scalable polyketide biosynthesis ${ }^{6}$. Informed by the in vitro reconstitution experiments summarized above, we engineered three plasmids with compatible antibiotic resistance markers and origins of replication that collectively encode the pathway. Plasmid pCKKPY222 encoded modules 1-2 as one bimodular protein and module 3. Plasmid pCK-KPY259 encoded module 4$\mathrm{KS}_{5}$ and the intact NOCAP_PKS2, and pCK-KPY178 encoded the $t$ AT-TEII and MBP-module $\mathrm{X}$ proteins (Figures 3b, S17). To enhance the malonyl-CoA pool in E. coli, pCK-KPY178 also encodes MatB and the Rhizobium leguminosarum malonate carrier protein Mat $\mathrm{C}^{18,19}$. Gratifyingly, E. coli BAP1[pCK-KPY222/pCK-KPY259/pCK-KPY178] produced 1 and 2 (Figures 3c, S18-23). E. coli-derived 1 and $\mathbf{2}$ had the same MS/MS fragments as $\mathbf{1}$ and $\mathbf{2}$ produced in vitro. Derivatization of $\mathbf{1}$ and $\mathbf{2}$ with Girard's reagent $\mathrm{T}^{20}$ confirmed the presence of an aldehyde (Figures S24-27). 3 and 4 were much lower in abundance from extracts of this strain, suggesting that these metabolites do not arise under physiological conditions, but are only synthesized under conditions with excess substrates. Because of their scarcity in $E$. coli, 3 and $\mathbf{4}$ were not further characterized.

We isolated $\mathbf{1}$ and $\mathbf{2}$ as faint yellow solids from $4 \mathrm{~L}$ of $E$. coli BAP1[pCK-KPY222/pCK-KPY259/pCK-KPY178] using lipid extraction with methyl tert-butyl ether/methanol ${ }^{21}$, $\mathrm{C}_{18}$ solid-phase extraction and UV-absorbance-guided semi-preparative HPLC (Figures S28-30). A number of $1 \mathrm{D}$ - and $2 \mathrm{D}-\mathrm{NMR}$ experiments ( ${ }^{1} \mathrm{H}, \mathrm{COSY}$, TOCSY, HSQC, HMBC, NOESY and ROESY) allowed us to fully elucidate their chemical structures (Figures 4, S31-62; Table S2). For 1, COSY, TOCSY and HMBC experiments established carbon-carbon connectivity from C-1 (195.1 ppm) to C-22 (18.3 ppm) as well as the phenolic moiety resulting from $\mathrm{C}-2-\mathrm{C}-7$ aldol condensation. These spectra also revealed a pair of conjugated trienes, one synthesized by modules 1-3 

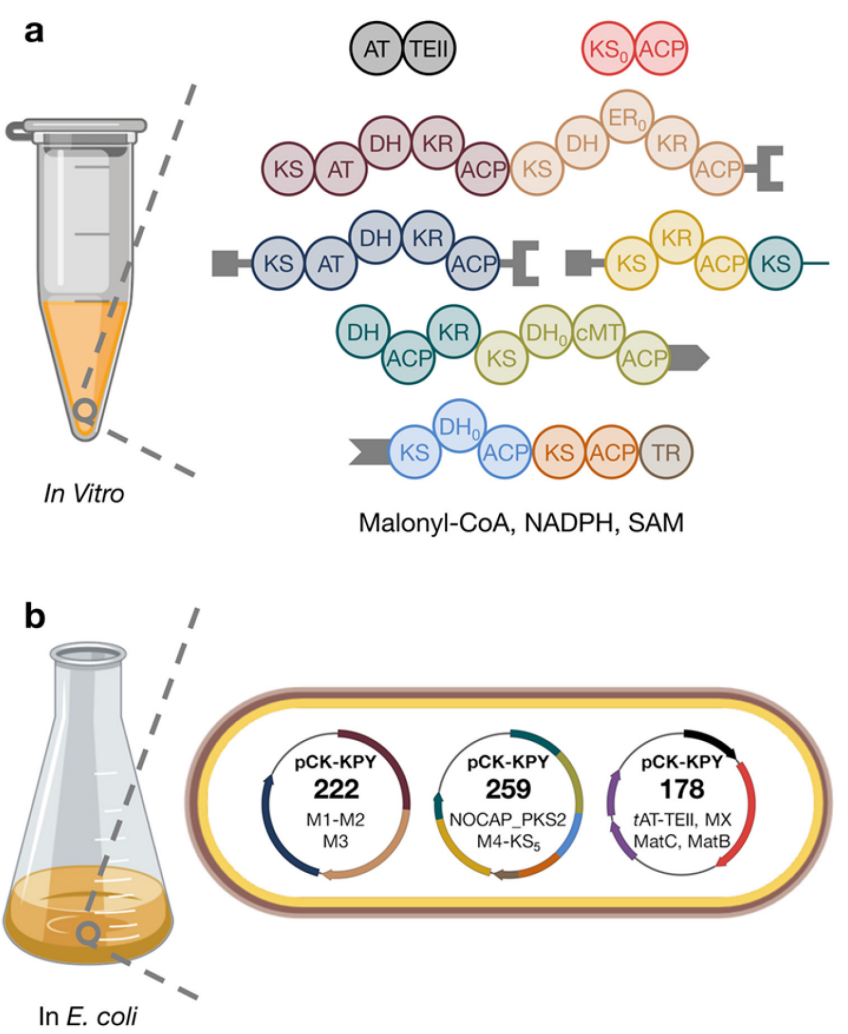

c
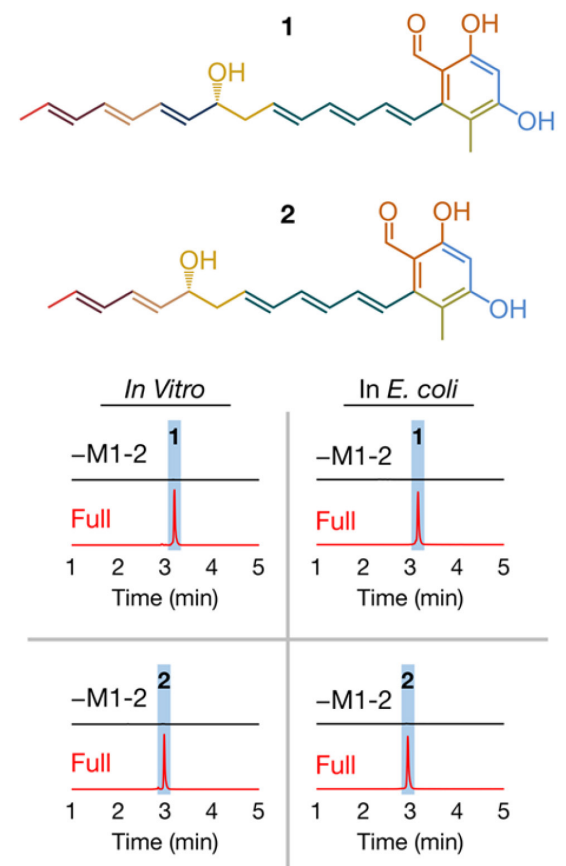

Figure 3. Reconstitution of the NOCAP synthase (a) in vitro and (b) in E. coli. (c) EICs of $\mathbf{1}$ and $\mathbf{2}$ from either in vitro reactions or E. coli pellet extracts. As a negative control, modules 1 and 2 (as one bimodular protein) were omitted.

and the other by module 5 . The aldehyde substituent at C-1 shows that $\mathbf{1}$ and $\mathbf{2}$ were released from the assembly line by the terminal TR domain. We observed the expected hydroxyl substituents at $\mathrm{C}-3$ (163.6 ppm, module 8), C-5 (16o.8 ppm, module 7) and C-15 (71.9 ppm, module 4), a singlet methyl substituent at C-6 (114.7 ppm, module
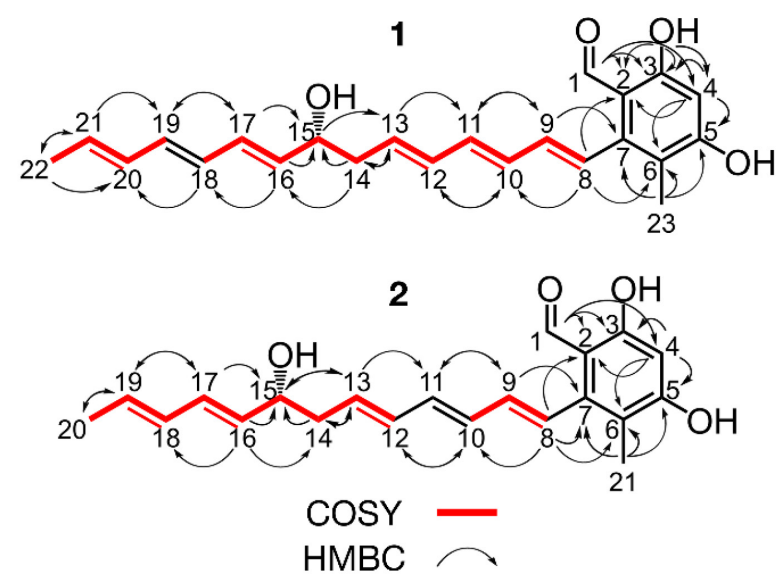

Figure 4. Structures of 1 and 2 assembled from $2 \mathrm{D}$ NMR data.

6) and a terminal doublet methyl (C-22 for 1, C-20 for 2, module X). ROESY analysis of $\mathbf{1}$ and NOESY analysis of $\mathbf{2}$ verified that all of their double bonds have trans stereoconfigurations as predicted by bioinformatic analysis ${ }^{22}$. To determine the absolute configuration of the stereocenter set by the KR domain of module 4, we converted the C-15 hydroxyl substituent of $\mathbf{2}$ to a Mosher ester ${ }^{23}$. Mosher ester analysis with COSY confirmed that the absolute configuration at $\mathrm{C}-15$ is $R$, also as predicted by bioinformatic analysis ${ }^{22}$ (Figures S63-66). Unlike 1, compound 2 featured a conjugated diene - not triene - in its "tail". We therefore hypothesized that a combination of the dissociated-by-design nature of module 3 and the broad substrate tolerance of the KS domain of module $4^{5}$ permitted facile chain translocation of the growing polyketide chain from module 2 to module 4 . Indeed, $E$. coli that does not express module 3 only produced 2, substantiating our hypothesis that the biosynthesis of $\mathbf{2}$ involves bypassing module 3 (Figure S67). Collectively, these spectroscopic efforts validated $\mathbf{l}$ as the aglycone product of the NOCAP synthase.

This report represents two milestones. First, we describe for the first time the full reconstitution (both in vitro as well as in E. coli) of an assembly-line PKS that is predominantly comprised of trans-AT modules. trans-AT PKSs represent over $23 \%$ of all sequenced assembly-line PKSs according to a recent survey ${ }^{24}$ and display remarkable architectural diversity; however, the understanding of trans-AT PKSs has significantly lagged that of cis-AT $\mathrm{PKSs}^{2}$. Based on this report, the NOCAP synthase is a strong contender as a model trans-AT PKS in the same vein as DEBS has been for cis-AT PKSs. Second, this work concludes the first example of natural product discovery by reconstituting orphan assembly-line PKSs in vitro or in E. coli. In principle, the methodology described here could be applied to other orphan polyketide natural products, especially those synthesized in low abundance or from unculturable organisms ${ }^{25}$. The discovery and structure elucidation of $\mathbf{1}$ will also allow us to turn our attention to the tailoring enzymes clustered with the NOCAP synthase genes and the ultimate characterization 

available under aCC-BY 4.0 International license.

of the biological role of its fully decorated natural product. Such efforts are compellingly motivated by the statistically significant but nonetheless correlative occurrence of this PKS only in strains associated with clinical cases of nocardiosis.

\section{AUTHOR INFORMATION}

\section{Corresponding Author}

*khosla@stanford.edu

\section{Present Addresses}

\#Department of Systems Biology, Blavatnik Institute at Harvard Medical School, Boston, MA 02115 and Wyss Institute for Biologically Inspired Engineering, Harvard University, Boston, MA 02115

\section{Author Contributions}

${ }^{\perp}$ C.W.L. and S.R.L. contributed equally.

\section{Notes}

The authors declare no competing financial interests.

\section{ACKNOWLEDGMENT}

We thank past and present members of the C.K. laboratory for fruitful discussions and suggestions. We also thank Theresa McLaughlin (Stanford University) and Jeffrey G. Pelton (University of California, Berkeley) for technical assistance, and Prof. Joseph D. Puglisi for access to his $500 \mathrm{MHz}$ NMR spectrometer. This work was supported by National Institutes of Health (NIH) Grant Ro1 GMo87934 (to C.K.) and NIH Grant F32 GM123637 (to K.P.Y.). This work utilized the Stanford Cancer Institute Proteomics/Mass Spectrometry Shared Resource, which is supported by NIH Grant $\mathrm{P}_{30}$ CA124435, and the $900 \mathrm{MHz}$ NMR spectrometer (funded by NIH Grant P41 GMo68933) at the Central California 9oo MHz NMR Facility.

\section{REFERENCES}

1. O'Brien, R. V.; Davis, R. W.; Khosla, C.; Hillenmeyer, M. E. Computational identification and analysis of orphan assemblyline polyketide synthases. J. Antibiot. 2o14, 67, 89.

2. Helfrich, E. J. N.; Piel, J. Biosynthesis of polyketides by trans-AT polyketide synthases. Nat. Prod. Rep. 2016, 33, 231.

3. Kageyama, A.; Yazawa, K.; Mukai, A.; Kohara, T.; Nishimura, K.; Kroppenstedt, R. M.; Mikami, Y. Nocardia araoensis sp. nov. and Nocardia pneumoniae sp. nov., isolated from patients in Japan. Int. J. Syst. Evol. Microbiol. 2004, 54, 2025.

4. Wilson, J. W. Nocardiosis: updates and clinical overview. Mayo Clin. Proc. 2012, 87, 403.

5. Kuo, J.; Lynch, S. R.; Liu, C. W.; Xiao, X.; Khosla, C. Partial in vitro reconstitution of an orphan polyketide synthase associated with clinical cases of nocardiosis. ACS Chem. Biol. 2016, 11, 2636.

6. Pfeifer, B. A.; Admiraal, S. J.; Gramajo, H.; Cane, D. E.; Khosla, C. Biosynthesis of complex polyketides in a metabolically engineered strain of E. coli. Science 2001, 291, 1790.

7. Lowry, B.; Robbins, T.; Weng, C. H.; O’Brien, R. V.; Cane, D. E.; Khosla, C. In vitro reconstitution and analysis of the 6deoxyerythronolide B synthase. J. Am. Chem. Soc. 2013, 135, 16809.

8. Olano, C.; Wilkinson, B.; Sanchez, C.; Moss, S. J.; Sheridan, R.; Math, V.; Weston, A. J.; Brana, A. F.; Martin, C. J.; Oliynyk, M.; Mendez, C.; Leadlay, P. F.; Salas, J. A. Biosynthesis of the angiogenesis inhibitor borrelidin by Streptomyces parvulus Tü4055: cluster analysis and assignment of functions. Chem. Biol. 2004, 11,87 .

9. Weber, T.; Laiple, K. J.; Pross, E. K.; Textor, A.; Grond, S.; Welzel, K.; Pelzer, S.; Vente, A.; Wohlleben, W. Molecular analysis of the kirromycin biosynthetic gene cluster revealed betaalanine as precursor of the pyridone moiety. Chem. Biol. 2008, 15,175 .

10. Gomez-Escribano, J. P.; Song, L. J.; Fox, D. J.; Yeo, V.; Bibb, M. J.; Challis, G. L. Structure and biosynthesis of the unusual polyketide alkaloid coelimycin $\mathrm{P} 1$, a metabolic product of the $c p k$ gene cluster of Streptomyces coelicolor M145. Chem. Sci. 2012, 3 , 2716.

11. Quadri, L. E.; Weinreb, P. H.; Lei, M.; Nakano, M. M.; Zuber, P.; Walsh, C. T. Characterization of Sfp, a Bacillus subtilis phosphopantetheinyl transferase for peptidyl carrier protein domains in peptide synthetases. Biochemistry 1998, 37, 1585.

12. Bisang, C.; Long, P. F.; Cortés, J.; Westcott, J.; Crosby, J.; Matharu, A. L.; Cox, R. J.; Simpson, T. J.; Staunton, J.; Leadlay, P. F. A chain initiation factor common to both modular and aromatic polyketide synthases. Nature 1999, 401, 502.

13. Dorrestein, P. C.; Bumpus, S. B.; Calderone, C. T.; GarneauTsodikova, S.; Aron, Z. D.; Straight, P. D.; Kolter, R.; Walsh, C. T.; Kelleher, N. L. Facile detection of acyl and peptidyl intermediates on thiotemplate carrier domains via phosphopantetheinyl elimination reactions during tandem mass spectrometry. Biochemistry 2006, 45, 12756.

14. Hughes, A. J.; Keatinge-Clay, A. Enzymatic extender unit generation for in vitro polyketide synthase reactions: structural and functional showcasing of Streptomyces coelicolor MatB. Chem. Biol. 2011, 18, 165.

15. Hu, Z.; Pfeifer, B. A.; Chao, E.; Murli, S.; Kealey, J.; Carney, J. R.; Ashley, G.; Khosla, C.; Hutchinson, C. R. A specific role of the Saccharopolyspora erythraea thioesterase II gene in the function of modular polyketide synthases. Microbiology 2003, 149, 2213.

16. Wu, N.; Cane, D. E.; Khosla, C. Quantitative analysis of the relative contributions of donor acyl carrier proteins, acceptor ketosynthases, and linker regions to intermodular transfer of intermediates in hybrid polyketide synthases. Biochemistry 2002, 41, 5056 .

17. Klaus, M.; Ostrowski, M. P.; Austerjost, J.; Robbins, T.; Lowry, B.; Cane, D. E.; Khosla, C. Protein-protein interactions, not substrate recognition, dominate the turnover of chimeric assembly line polyketide synthases. J. Biol. Chem. 2016, 291, 16404.

18. Lombó, F.; Pfeifer, B.; Leaf, T.; Ou, S.; Kim, Y. S.; Cane, D. E.; Licari, P.; Khosla, C. Enhancing the atom economy of polyketide biosynthetic processes through metabolic engineering. Biotechnol. Prog. 2001, 17, 612.

19. Leonard, E.; Yan, Y.; Fowler, Z. L.; Li, Z.; Lim, C. G.; Lim, K. H.; Koffas, M. A. Strain improvement of recombinant Escherichia coli for efficient production of plant flavonoids. Mol. Pharm. 2008, 5, 257.

20. Girard, A.; Sandulesco, G. Sur une nouvelle série de réactifs du groupe carbonyle, leur utilisation à l'extraction des substances cétoniques et à la caractérisation microchimique des aldéhydes et cétones. Helv. Chim. Acta 1936, 19, 1095.

21. Matyash, V.; Liebisch, G.; Kurzchalia, T. V.; Shevchenko, A.; Schwudke, D. Lipid extraction by methyl-tert-butyl ether for high-throughput lipidomics. J. Lipid Res. 2oo8, 49, 1137.

22. Caffrey, P. Conserved amino acid residues correlating with ketoreductase stereospecificity in modular polyketide synthases. Chembiochem 2003, 4, 654 .

23. Hoye, T. R.; Jeffrey, C. S.; Shao, F. Mosher ester analysis for the determination of absolute configuration of stereogenic (chiral) carbinol carbons. Nat. Protoc. 2007, 2, 2451. 
bioRxiv preprint doi: https://doi.org/10.1101/2020.01.28.923383; this version posted January 29, 2020. The copyright holder for this preprint (which was not certified by peer review) is the author/funder, who has granted bioRxiv a license to display the preprint in perpetuity. It is made available under aCC-BY 4.0 International license.

24. Nivina, A.; Yuet, K. P.; Hsu, J.; Khosla, C. Evolution and diversity of assembly-line polyketide synthases. Chem. Rev. 2019, 119, 12524.

25. Rutledge, P. J.; Challis, G. L. Discovery of microbial natural products by activation of silent biosynthetic gene clusters. Nat. Rev. Microbiol. 2015, 13, 509. 